\title{
HERRAMIENTA PARA MEDICIÓN Y ANÁLISIS DEL RECORRIDO DEL TRABAJO EN BODEGAS DE GESTIÓN DE MUESTRAS
}

\section{Tool for measuring and analyzing the work route in sample management warehouses}

\author{
Manuel Arturo Jiménez Ramírez ${ }^{1}$ \\ ${ }^{1}$ Universidad Pontificia Bolivariana \\ Email: manuel.jimenezr@upb.edu.co
}

(Recibido Febrero 10 de 2019 y aceptado Mayo 22 de 2019)

\section{Resumen}

El presente artículo muestra la estructuración y método de una herramienta de medición y recorrido del trabajo realizado en la bodega de reactivos y la bodega de muestras en custodia de una empresa de Oil y Gas en Colombia. Esta herramienta tiene dos propósitos: el primero, es el de describir las tareas realizadas y el segundo, proporcionar un esquema para el desarrollo y mejoramiento de los procesos en el Centro de recepción de muestras de un Centro de Investigación y desarrollo del sector de Oil y Gas, en adelante CEMIM. La medición del trabajo es un método de investigación basado en la aplicación de diversas técnicas para determinar las actividades y el contenido de un trabajo definido, fijando el tiempo que tarda un trabajador calificado en realizar la tarea de acuerdo con un rendimiento estandarizado preestablecido. Con base en lo anterior, se realizó un estudio del trabajo en las bodegas del CEMIM de acuerdo con el número de estanterías, y el recorrido realizado en estas. Los resultados muestran que, al considerar explícitamente los patrones de recorrido realizados por el operario, el análisis de la herramienta estructurada proporciona ventajas relacionadas con la descripción de las tareas y el desarrollo de métodos para el mejoramiento de los procesos.

Palabras clave: descripción de las tareas, esquema de desarrollo, estructuración y método, estudio del trabajo.

\begin{abstract}
The present paper shows the organization and methods of a work-and-route measurement tool carried out in the warehouse of reactive samples and in the warehouse of samples in custody of an Oil and Gas company in Colombia. This tool is twofold: first is to describe the task carried out and second, is to provide a scheme for the development and improvement processes in the Center of Investigation and development of Oil and Gas Sector, henceforth it is known as CEMIM. The measurement of this work is a research method based on the application of several techniques to determine the activities and content about a defined job, fixing the time in which a qualified worker takes to accomplish the task according to a pre-established standardized performance. Based on this, a work study was carried out at the CEMIM warehouses in accordance to the number of shelves, and the route made by themselves. The results show that when you are considering the route of patterns made by the worker, the analysis of the structured tool provides advantages related to the descrip-tion of the task and the development of methods for the improvement of processes.
\end{abstract}

Key words: development patterns, organization and methods, task analysis, work study. 


\section{INTRODUCCIÓN}

Este artículo presenta el desarrollo y aplicación de una herramienta de medición y recorrido del trabajo realizado en el Centro de Manejo Integral de Muestras (CEMIM). EI CEMIM nació, principalmente, como una pieza importante en la cadena de calidad y logística entre los diferentes clientes y la sistematización de los laboratorios, ya sea para la prestación de servicios técnicos especializados o en el desarrollo de proyectos de investigación que adelanta el Instituto Colombiano de petróleos (ICP) para una empresa del sector Oil y Gas en Colombia y terceros.

Dentro estas estrategias para adelantar investigación aplicada se encuentran los acuerdos de cooperación entre la Universidad Pontificia Bolivariana (UPB) y el ICP; y esta investigación hace parte del Acuerdo de Cooperación No. 07 para implementar y evaluar el plan de mejoramiento de los procesos de manejo integral de muestras del CEMIM, derivados del Acuerdo No. 002 UPB-ICP.

En este sentido, dada la pertinencia que tiene para el ICP, el CEMIM, en función a su mejoramiento continuo, adelanta propuestas de diseño y aplicación de herramientas de medición, conducentes a la mejora de la productividad.

El enfoque metodológico, se constituye como la particularidad central de este estudio, el cual culmina con la conformación, construcción de conceptos y variables con la mira puesta en la aplicación dentro del CEMIM, con el fin de ofrecerle una herramienta de medición del trabajo que se desempeña en las bodegas de: reac-tivos y muestras en custodia. El artículo finaliza con ejemplos sobre la aplicación de la herramienta y la explicación de cada una de las variables que la componen.

Este artículo está divido en tres secciones, a parte de esta introducción. La sección siguiente presenta las nociones del estudio de trabajo y herramientas de medición, la sección tercera expone la metodología y el método utilizado, y la cuarta sección se ocupa de la conformación y construcción del modelo; se presentan ejemplos de aplicación y una nota aclaratoria para la herramienta de la bodega de muestras en custodia. Por último, el documento culmina con las conclusiones sobre los resultados más importantes.

\section{REVISIÓN DE LITERATURA}

El estudio de trabajo es una técnica que se aplica en empresas de diferentes sectores en las que el objetivo es medir los movimientos y los procesos de la trayectoria, medir el tiempo en que se realiza una tarea y analizar el diseño de la estación de trabajo para establecer mejores formas de realizar un trabajo. Actualmente, las empresas centran sus esfuerzos en maximizar sus indicadores de productividad, y por esta razón se vuelve importante el diseño de herramientas y mecanismos para medir y estandarizar las actividades llevadas a cabo en la empresa.

De acuerdo con lo anterior, [1] define la medición del trabajo como un método de investigación basado en la aplicación de diversas técnicas para determinar las actividades y el contenido de un trabajo definido, fijando el tiempo que tarda un trabajador calificado en realizar la tarea de acuerdo con un rendimiento estandarizado preestablecido.

Sobre la base de la mejora de los indicadores de productividad, [2] se estableció que el estudio de los movimientos ofrece un gran potencial de ahorro en cualquier empresa humana. Esto significa que se podría aho-rrar el costo de un trabajo eliminándolo, esto si el estudio de trabajo concluye que la actividad no es necesaria y no agrega valor al proceso. En [2] se mencionan las siguientes ventajas: (1) se puede reducir en gran medida combinando elementos de una tarea con elementos de otra tarea; (2) puede reorganizar los elementos de una tarea para facilitar el proceso del camino; (3) también puede simplificar la tarea colocando componentes y herramientas cerca del punto de uso, colocando componentes y herramientas por adelantado, proporcionando asistencia mecánica o reduciendo los elementos del trabajo para que consuman menos tiempo; (4) incluso puede solicitar que un componente se rediseñe para facilitar sus producciones; (5) en la reducción de costos, la simplificación es el 
procedimiento que requiere más tiempo, además de quesu ahorro es pequeño si se compara con la eliminación y combinación de elementos, pero siempre puede simplificarse.

Un poco de historia sobre cómo surgió el estudio de trabajo se pudo encontrar bajo el nombre de "Adminis-tración Científica" y fue Frederick Taylor el pionero de este desarrollo y dio las bases del sistema de medición de trabajo actual. La Administración Científica se basa en la racionalización de la labor del trabajador con miras a aumentar la productividad [3]. Hay evidencia de que, en Colombia, a partir de 1912, se difundió y se aplicó a pesar del incipiente desarrollo industrial [3].

Desde un punto de vista, es comprensible que la Administración Científica como Sistema de Gestión sea base en el Paradigma Cartesiano, dividiendo las actividades (sistema) en pequeñas partes para comprender su comportamiento. Las ideas principales de la Administración Científica consistieron en descomponer el proceso de producción en sus componentes y mejorar cada eficiencia; en esencia, Taylor estaba tratando de es-tandarizar las unidades de trabajo y hacerlas intercambiables [4]. Además, más que simplemente medir y comparar la tasa de trabajo de los hombres, Taylor buscó implacablemente la mejor manera de hacer un trabajo: los manuales se ajustaron para maximizar la eficiencia al analizar cada componente por separado y eliminar todos los movimientos falsos, lentos, o sin utilidad; los trabajos mecánicos se aceleraron a través de hojas de cálculo, accesorios y otros dispositivos, muchos inventados por él mismo [4].

El estudio de tiempo y la estandarización [5] es una técnica de medición útil para evaluar los contenidos de trabajo y para optimizar los sistemas de producción tanto prospectivos (antes del inicio de la producción) como durante una producción en ejecución. En este sentido, la estandarización [6] ocupa el segundo lugar, por medios materiales, entre los elementos que determinaron el éxito comercial de una empresa porque contienen especificaciones técnicas u otros acuerdos documentados que pueden reutilizarse ampliamente como reglas, directrices o definiciones de las características, con el fin de garantizar que el producto sea apropiado para el propósito que fue creado.

Una de las técnicas utilizadas en el estudio de trabajo es el diagrama de flujo. Esta técnica ayuda a visualizar los desplazamientos dentro de un proceso [7, 8]. En [7] esta técnica se usa fundamentalmente para investigar la secuencia de pasos (todos o algunos de los procesos) en la elaboración de una pieza para indicar qué se debe hacer y para eliminar los reprocesos y, por lo tanto, encontrar la forma más económica de procesar. En [8] se usa el diagrama de flujo secuencial para mostrar los pasos discretos en un proceso secuencialmente en el orden en que se realiza la tarea, y porque se organiza la información de la tarea de forma esquemática.

Los diagramas de flujo pasan a formar parte de la planificación del diseño que representa la disposición física de los objetos en el espacio [9]. Los objetivos más comunes de los problemas de diseño son la minimización de los costos de transporte de las materias primas, piezas, herramientas, productos en proceso y productos terminados entre las instalaciones, lo que facilita el flujo de tráfico y minimiza los costos de esta [9-11].

Basado en el análisis logístico de material (ajuste de diseño de celular) [12], se estudian algunas tecnologías clave que orientan el diseño de la línea de producción celular desde el punto de vista de las siguientes aplicaciones: (1) los patrones logísticos de material están modularizados, (2) en los estadísticos de logística se presentan las tasas de flujo en los principales canales de transporte y entre las máquinas, y (3) las medidas de ajuste de la disposición, incluyendo la adición de equipos, la sustitución de equipos, la compartición de equipos y el ajuste de la carcasa bajo el objetivo general de minimizar el costo logístico.

\section{METODOLOGÍA}

Este proyecto de investigación es de tipo proyectivo, porque su propósito es el de proponer 
al Centro de Manejo Integral de Muestras del Instituto Colombiano de Petróleos una herramienta de medición del recorrido y trabajo realizado en las bodegas de almacenamiento de muestras; es de tipo exploratorio y descriptivo porque se realizará una descripción de los procesos y diseño de una herramienta de medición de trabajo y recorrido [13]. Todo esto es realizado a través de una fase exploratoria de recolección de información proporcionada por los trabajadores del CEMIM y de revisión de literatura.

\subsection{El método}

Este estudio fue llevado a cabo en el Centro Integral de Muestras en dos bodegas de almacenamiento: la bodega de custodia y la bodega de reactivos. El objetivo de este estudio es el de proporcionar una herramienta al CEMIM para entender la forma en la cual las bodegas son utilizadas durante los procesos de Gestión integral de muestras y Gestión de inventarios y almacén, de tal forma que la información permita identificar patrones para diseñar un circuito más amigable para el trabajador y eficiente para el CEMIM en futuras investigaciones.

\subsection{Fase exploratoria}

En esta fase se aplicó la técnica del interrogatorio a los trabajadores del CEMIM para recolectar información relacionada con los procesos de Gestión integral de muestras y Gestión de inventarios y almacén; todo esto acompañado del mismo recorrido de las actividades realizadas.

Las bodegas de custodia y la bodega de reac-tivos fueron visitadas en la que información específica como el número de estanterías, módulos por estanterías, identificación de pasillos y ubicación en relación con la puerta de ingreso fueron tenidos en cuenta.

También se revisaron documentos de investigaciones realizadas anteriormente, sobre la definición del CEMIM como parte del Instituto Colombiano de Petróleos.

\subsection{Desarrollo hojas de recorrido}

Todos los autores de este documento son expertos en temas de medición del trabajo y han dado validez a este formato ya que cumple con las necesidades del análisis de los procesos en el CEMIM. Adicionalmente, este documento fue inspirado por el trabajo de [14]. En su investigación, los autores diseñaron una técnica para realizar un estudio de tiempos y movimientos en cocinas.

Al probar la técnica, los análisis de resultados demostraron que el formato provee la misma información que un diagrama de flujo y adicionalmente, la lectura de la información es más fluida y entendible [14].

\section{RESULTADOS}

Para la técnica del interrogatorio se diseñaron una serie preguntas relacionadas con el trabajo realizado en el CEMIM, desde que se reciben las solicitudes por parte de los clientes hasta que son procesadas y almacenadas.

\subsection{Centro de manejo integral de muestras (CEMIM).}

EI CEMIM hace parte del Instituto Colombiano de Petróleos, con sede en Piedecuesta, Santander, donde se desarrolla, adapta y transfiere soluciones tecnológicas de alto impacto para la industria petrolera, fortale-ciendo el sistema de competitividad e innovación del país [15].

El CEMIM es el área que hace parte del Departamento de Servicio Técnico de Laboratorios de Transporte y Transversal, y brinda soporte transversal a todos los laboratorios, plantas piloto y proyectos aplicados en el ICP [15]

El CEMIM es una pieza importante en la cadena de calidad y logística entre los diferentes clientes y la sistematización de los laboratorios, ya sea para la prestación de servicios técnicos especializados o en el desarrollo de proyectos de investigación que adelanta el ICP junto con la empresa y terceros [16].

\subsection{Procesos realizados en el CEMIM}

Los resultados muestran que en el CEMIM se realizan dos macro-procesos: Gestión Integral de Muestras y Gestión de Inventarios y Almacén. 
En la Gestión Integral de Muestras, se realiza la recepción de la muestra y la validación del estado de esta, seguido por el almacenamiento temporal de las mismas, su generación de identificación de cada una en el sistema de información, el fraccionamiento y etiquetado, la entrega de muestras respectivas al laboratorio y el ingreso de datos tomados en campos.

Por otro lado, la Gestión de Inventarios y Almacén, se realiza con las muestras en custodia-ingreso, la gestión de muestra en custodia-devolución al cliente en caso de que se requiera nuevamente para análisis, la gestión de muestras en custodia-monitoreo; y, por último, se realiza la gestión de muestras en custodia-eliminación que consiste en comunicarse con el dueño de la muestra y gestionar la eliminación de las que no se vuelven a utilizar.

\subsection{Desarrollo herramienta de medición y recorrido}

\subsubsection{Esquematización}

Con las visitas realizadas el CEMIM, se tomó registro fotográfico de las bodegas para el diseño de tablas que permitan registrar los movimientos y traslados realizados dentro de las bodegas, tanto al almacenamiento como operaciones de recolección para entrega de muestras.
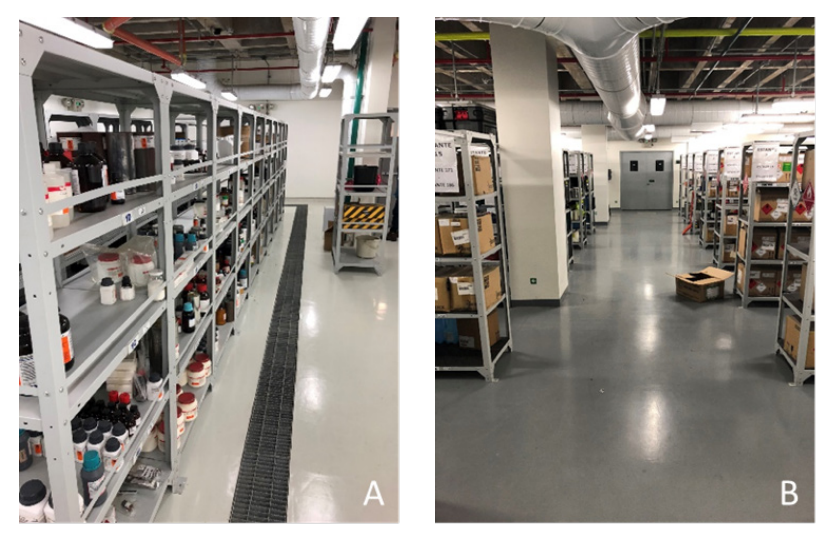

Figura 1. Bodega de reactivos. (A) Bodega de custodia. (B).

Con la visita y registro fotográfico se realizó un esquema de vista superior de las bodegas para tener una visión global.

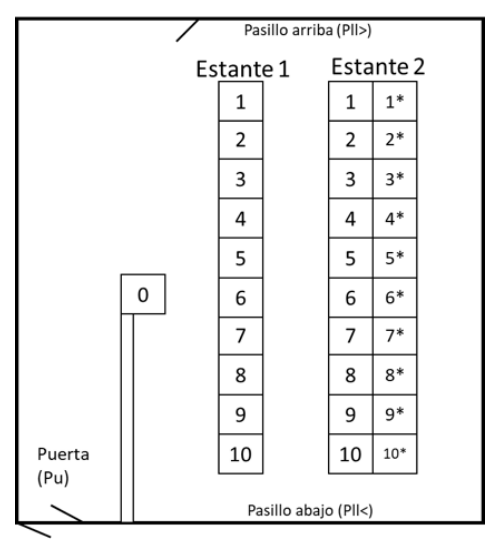

Figura 2. Esquema vista superior Bodega de reactivos.

En la figura 2 se presenta el esquema de la bodega de reactivos, y se observa que esta bodega posee dos estanterías, cada estantería con 10 módulos, y 4 espacios verticales para almacenamiento de reactivos. El estante dos tiene dos estantes unidos, realizando la diferencia con el asterisco que acompaña el número. Se identificaron, adicionalmente, las variables "Puerta" puesto que el recorrido del trabajo inicia desde el ingreso a la bodega y finaliza en este mismo punto; y la variable "Pasillo" para conocer si la visita a los módulos en los estantes fue realizada por el pasillo de arriba o el pasillo de abajo.

Se observa también un estante con el número cero (0), esto quiere decir que es un estante con un único módulo y 4 espacios verticales; pero para la herramienta diseñada no se tuvo en cuenta porque en este estante no se almacenan reactivos.

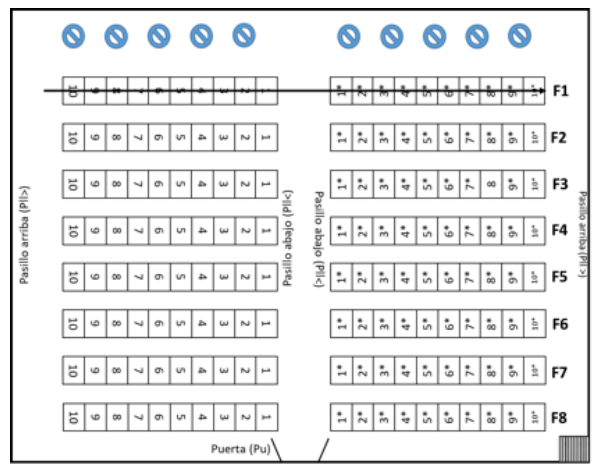

Figura 3. Esquema vista superior Bodega de custodia. 
En la figura 3 se muestra el esquema de la bodega de custodia, y se aprecia que esta es más grande que la bodega de reactivos. Esta bodega cuenta con 8 filas de estanterías (a mano izquierda y mano derecha), separados por un pasillo central. Cada estante posee 10 módulos con espacios verticales para almacenamiento de muestras de custodia. También se identificaron las variables "Pasi"lo" para conocer si el recorrido del trabajo se realizó por la parte de arriba o la parte de debajo de las bodegas. Adicionalmente, la variable "Puerta" también fue necesario involucrarla por las mismas razones que se identificó en la bodega de reactivos. El trabajo inicia desde el ingreso por la puerta y finaliza en este mismo punto.

\subsubsection{Diseño de herramienta de medición y recorrido}

Con base en el esquema de conocimiento de los procesos realizados en el CEMIM, el registro fotográfico y el esquema de vista superior se diseñaron las siguientes herramientas para la bodega de reactivos (Tabla 1) y de custodia (Tabla 2) con el propósito de conocer en las operaciones realizadas, el tiempo en el que se realiza y el desplazamiento realizado, así como identificar cuál fue el quipo utilizado para desempeñar la labor de almacenar o retirar muestras de las bodegas.

La herramienta permite conocer el desplazamiento $y$ el trabajo desempeñado que realiza un trabajador des-de la entrada hasta la salida de la bodega. Las columnas representan las variables identificadas según el esquema de vista superior de la bodega (Figura 2). La definición de las variables es la siguiente:

Las primeras 8 columnas indican los movi-
mientos realizados en el espacio de trabajo.

Pu: Puerta (ingreso y salida)

Est1: Estantería 1

Est2: Estantería 2

Pll>: Parte superior del pasillo
PIl<: Parte inferior del pasillo

AE: permite registrar si la operación se realiza fuera del área de trabajo.

DE: esta variable registra movimientos posibles debido al equipo utilizado que no permita atravesar los pasillos.

BP: pequeña bodega en la que se realizó parte del proceso.

Las siguientes columnas tienen como objetivo recolectar información y frecuencias del trabajo realizado, la hora en la que se realizó la actividad, el equipo utilizado, y se añade una columna de observaciones por si se hace necesario especificar cuál fue la labor desempeñada. Las variables de estas columnas son:

O: Operación; T: Transporte; D: Demora y; A: Almacenamiento

ModEst: variable que se debe diligenciar indicado en número de módulo de la estantería que fue visitada como parte del desplazamiento en el proceso.

Tiempo: permite registrar el tiempo en que se realizó el proceso.

Equipo: registra el equipo utilizado para llevar a cabo la operación.

Un ejemplo de la utilización de la tabla es el que se muestra a continuación (Ver Tabla 1). En este recorrido se almacenaron reactivos en los dos estantes y en módulos diferentes. Esto es debido a que los reactivos poseen restricciones de cercanía y peligrosidad.

El trabajo desempeñado inicia desde el ingreso por la puerta, el trabajador lleva consigo un carro transportador y se dirige al módulo 10 del segundo estante y desempeña el trabajo de almacenar los reactivos; este recorrido lo realiza desplazándose primero al pasillo de la parte inferior de la bodega. 
Tabla 1. Ejemplo medición y recorrido del trabajo bodega de reactivos

\begin{tabular}{|c|c|c|c|c|c|c|c|c|c|c|c|c|c|c|c|}
\hline \multirow{2}{*}{\multicolumn{3}{|c|}{$\begin{array}{l}\text { Fecha: } \\
\text { Estudio: }\end{array}$}} & & & & & & & & & & Hoja: & 1 & de & 1 \\
\hline & & & \multicolumn{13}{|c|}{ Recorrido en bodega de reactivos } \\
\hline \multicolumn{3}{|c|}{ Equipo: } & \multicolumn{13}{|c|}{ Carrito Transportador (CT); Gancho (G); Cava (C) } \\
\hline $\mathrm{Pu}$ & Est1 & PII> & PII< & Est2 & $\mathrm{AE}$ & DE $S$ & sc & 0 & $\mathrm{~T}$ & D & A & ModEst & Tiempo & Equipo & Num.Ob. \\
\hline Pet & Est1 & $\mathrm{PII}>$ & $P \|<$ & Est2 & $\mathrm{AE}$ & $\mathrm{DE} S \mathrm{~S}$ & sc & 0 & $\mathrm{~T}$ & $\mathrm{D}$ & A & & $11: 25 \mathrm{am}$ & & 1 \\
\hline $\mathrm{Pu}$ & Est1 & $\mathrm{PII}>$ & Fोe & Est2 & $\mathrm{AE}$ & $\mathrm{DE} S \mathrm{~S}$ & sc & 0 & (T) & $\mathrm{D}$ & $\mathrm{A}$ & & $11: 25 a m$ & & 2 \\
\hline $\mathrm{Pu}$ & Est1 & $\mathrm{PII}>$ & $\mathrm{PII}<$ & Es2 & $\mathrm{AE}$ & $\mathrm{DE}$ & SC & 0 & (T) & $\mathrm{D}$ & $\mathrm{A}$ & $10^{*}$ & 11:26am & CT & 3 \\
\hline $\mathrm{Pu}$ & Est1 & $\mathrm{PII}>$ & 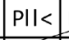 & $E \searrow^{\star}$ & $\mathrm{AE}$ & $\mathrm{DE}$ & sc & 0 & $\mathrm{~T}$ & $\mathrm{D}$ & (A) & $10^{*}$ & 11:27am & CT & 4 \\
\hline $\mathrm{Pu}$ & Est1 & Pest & $P \| \mid<$ & Est2 & $\mathrm{AE}$ & $\mathrm{DE}$ & sc & 0 & (T) & $\mathrm{D}$ & A & & 11:28am & & 5 \\
\hline $\mathrm{Pu}$ & Esti & $\mathrm{PII}>$ & $P \| \mid<$ & Est2 & $\mathrm{AE}$ & $\mathrm{DE} S$ & sc & 0 & (T) & $\mathrm{D}$ & $\mathrm{A}$ & 1 & $11: 29 \mathrm{am}$ & CT & 6 \\
\hline $\mathrm{Pu}$ & EX1 & $\mathrm{PII}>$ & $P \mid \mathrm{P}$ & Est2 & $\mathrm{AE}$ & $\mathrm{DE}$ S & SC & 0 & $\mathrm{~T}$ & $\mathrm{D}$ & (A) & 1 & 11:29am & CT & 7 \\
\hline Put & Est1 & $\mathrm{PII}>$ & $P \mid \mathrm{P}$ & Est2 & $\mathrm{AE}$ & $\mathrm{DE}$ S & sc & 0 & (T) & $\mathrm{D}$ & $\mathrm{A}$ & & 11:30am & & 8 \\
\hline \multirow[t]{2}{*}{$\mathrm{Pu}$} & Est1 & $\mathrm{PII}>$ & $P|l|$ & Est2 & $\mathrm{AE}$ & $\mathrm{DE}$ & sc & 0 & $\mathrm{~T}$ & $\mathrm{D}$ & $\mathrm{A}$ & & & & 9 \\
\hline & & & ecuen & cia \# & & & & 0 & 5 & 0 & 2 & & & & \\
\hline \multicolumn{16}{|c|}{ Observaciones } \\
\hline \multicolumn{16}{|l|}{1} \\
\hline \multicolumn{16}{|l|}{2} \\
\hline \multicolumn{16}{|l|}{3} \\
\hline 4 & \multicolumn{15}{|c|}{ Almacenamiento de reactivos } \\
\hline \multicolumn{16}{|l|}{5} \\
\hline \multicolumn{16}{|l|}{6} \\
\hline 7 & \multicolumn{15}{|c|}{ Almacenamiento de reactivos } \\
\hline \multicolumn{16}{|l|}{8} \\
\hline 9 & & & & & & & & & & & & & & & \\
\hline
\end{tabular}

Seguidamente, se devuelve al pasillo superior y almacena los reactivos en el primer módulo del primer estante. Como se muestra en la tabla, el recorrido, junto con el almacenamiento de reactivos, tuvo una duración de 5 minutos.

En relación con la bodega de muestras en custodia, se diseñó la siguiente herramienta (Tabla 2) que va a permitir, del mismo modo, que en la bodega de reactivos, se analice los desplazamientos realizados por los trabajadores, según la labor desempeñada en la bodega. Esta herramienta permite representar el desplazamiento realizado por el operario en la bodega de muestras en custodia. Las primeras 14 columnas representan las variables identificadas, de acuerdo con el esquema de vista superior de la bodega (Figura 3). La definición de las variables es la siguiente:

Do: Puerta (ingreso y salida)

F1, F2, F3, F4, F5, F6, F7, F8: Fila 1, 2, 3, 4, 5, 6, 7 y 8 n respectivamente, que contienen, cada una, dos estanterías divididas por el pasillo central (ver Figura 3).

Pll>: Parte superior de los pasillos, distinguidos por los módulos números 10 y 10*, según la dirección que seleccione el operario.

PIl<: Parte inferior del pasillo, representada por el pasillo central o por las áreas de los módulos de estante-rías 1 y $1 *$.

Pe: Atravesar el pasillo; implica un desplazamiento hacia las estanterías de la dirección opuesta. Esta variable no es requerida en el momento de trazar el recorrido, porque es entendido que si se coloca que el trabajador visitó el módulo 1 y luego el $1^{*}$ tuvo que cruzar el pasillo central.

Esta variable tiene un propósito a nivel prospectivo, es decir, puede suceder que el trabajo tenga que ser realizado en el Pasillo Central por varias razones; una de 
ellas puede ser las dimensiones del equipo utilizado para transportar las muestras. Sí el equipo utilizado es muy grande y no puede entrar a los pasillos, se debe dejar en el pasillo central mientras los trabajadores recolectan o almacenan las muestras y las van depositando en el equi-

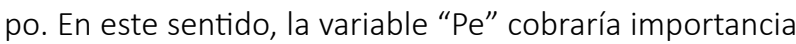
para indicar que el trabajo se desempeñó en este lugar.

Pueden suceder otro tipo de eventos como demoras que puedan suceder al intentar cruzar el pasillo, por cual se debería dejar indicado en el recorrido realizado.

Para evitar añadirle complejidad a esta herramienta, y que no surgieran un número de columnas que dificultaran trazar los recorridos, se pensó en filas de estantes, en donde la dirección tomada por el trabajador va a estar relacionada con el número asociado al módulo de los estantes.

Es decir, si el trabajador visitó la parte izquierda de la bodega se indicará en la herramienta con el número de módulo visitado del 1 al 10 (según la fila de estantería); ahora, si el trabajador visitó el lado derecho de la bodega, se indicará en la herramienta con el número de módulo de estantería del 1 al 10 acompañado de un asterisco (*).

Las siguientes columnas en la herramienta dan cuenta acerca de los espacios en los que se realizaron las actividades; es decir, si fueron realizados por fuera de las bodegas, o si el desplazamiento se realizó en algún otro punto de la bodega debido a los equipos. Las variables son las siguientes:

AO: permite registrar si la operación se realiza fuera del área de trabajo.

DE: esta variable registra movimientos posibles debido al equipo utilizado que no permita atravesar los pasillos.

Las siguientes columnas tienen como objetivo recolectar información y frecuencias del trabajo realizado, la hora en la que se realizó la actividad, el equipo utilizado, y se añade una columna de observaciones por si se hace necesario especificar cuál fue la labor desempeñada.
Las variables de estas columnas son:

O: Operación; T: Transporte; D: Demora y; S: Almacenamiento.

ModEst: variable que se debe diligenciar indicado en número de espacio en estantería que fue parte del desplazamiento en el proceso.

Tiempo: permite registrar el tiempo en que se realizó el proceso.

Equipo: registra el equipo utilizado para llevar a cabo el proceso.

Un ejemplo de la utilización de la tabla es el que se muestra a continuación (ver Tabla 2). El recorrido implica la recolección de reactivos de cloruro de paladio, peróxido de hidrógeno y un equipo de manejo de fluidos.

El trabajo desempeñado inicia con el ingreso por la puerta, luego el trabajador se dirige al lado derecho de la estantería y recoge el cloruro de paladio en el módulo 10; seguido, cruza el pasillo central y visita el módulo 7 donde recoge peróxido de hidrógeno, y, por último, el trabajador cruza nuevamente el pasillo y recoge la tecnología de manejo de fluidos, TEM. La duración del recorrido es 13 minutos.

Una nota aclaratoria con respecto al ingreso a la bodega de muestras en custodia. Como se ilustra en la Figura 3, al ingreso por la puerta, se observan directamente los pasillos identificados con las variables: $\mathrm{Pll}<\mathrm{y}$ Pe.

Al ingresar por la puerta, siendo este el primer desplazamiento, se puede omitir el punto (únicamente si el trabajo no se desempeñó en este punto) que indica que se desplazó al pasillo inferior dado que este espacio se debe cruzar para llegar al primer destino. Una vez, visitado el primer módulo de estantería ya se hace necesario indicar si el trabajador se desplazó por el pasillo de arriba o por el pasillo de abajo para llegar al siguiente punto en su recorrido. 
Tabla 2. Herramienta de medición y recorrido para bodega de muestras en custodia

\begin{tabular}{|c|c|c|c|c|c|c|c|c|c|c|c|c|c|c|c|c|c|c|c|c|c|}
\hline \multirow{2}{*}{\multicolumn{3}{|c|}{$\begin{array}{c}\text { Fecha: } \\
\text { Estudio: }\end{array}$}} & & & & & & & & & & & & & & \multicolumn{3}{|c|}{ Hoja: } & -1_ & de & -1 \\
\hline & & & \multicolumn{19}{|c|}{ Recorrido en bodega de custodia } \\
\hline \multicolumn{3}{|c|}{ Equipo: } & \multicolumn{19}{|c|}{ Carrito Transportador (CT); Gancho (G); Cava (C) } \\
\hline $\mathrm{Pu}$ & F1 & F2 & F3 & F4 & F5 & F6 & F7 & F8 & PII> & Pll< & $\mathrm{Cr}$ & AO & DE & 0 & $\mathrm{~T}$ & D & A 1 I & ModEst & Tiempo & Equipo & Num.Ob. \\
\hline$P$ & E1 & E2 & F3 & F4 & F5 & F6 & F7 & F8 & PII> & $\mathrm{PII}<$ & $\mathrm{Cr}$ & $\mathrm{AO}$ & $\mathrm{DE}$ & 0 & $\mathrm{~T}$ & D & $\mathrm{A}$ & & 10:38am & & 1 \\
\hline $\mathrm{Pu}$ & F1 & $\mathrm{F} 2$ & F3 & F4 & F5 & F6 & F7 & F8 & $P \mid>$ & $P$ & $\mathrm{Cr}$ & $\mathrm{AO}$ & $\mathrm{DE}$ & 0 & (I) & $\mathrm{D}$ & $\mathrm{A}$ & & 10:39am & & 2 \\
\hline $\mathrm{Pu}$ & $x$ & F2 & F3 & F4 & F5 & F6 & F7 & F8 & PII> & $P \|<$ & $\mathrm{Cr}$ & $\mathrm{AO}$ & DE & 00 & (T) & D & $\mathrm{A}$ & 10 & 10:39am & & 3 \\
\hline $\mathrm{Pu}$ & 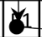 & $\mathrm{F} 2$ & F3 & F4 & F5 & F6 & F7 & F8 & 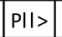 & $P \|<$ & $\mathrm{Cr}$ & $\mathrm{AO}$ & DE & (a) & $\mathrm{T}$ & $\mathrm{D}$ & $\mathrm{A}$ & 10 & 10:30am & & 4 \\
\hline $\mathrm{Pu}$ & F1 & F2 & F3 & F4 & F5 & F6 & F7 & F8 & PII) & $1<$ & $\mathrm{Cr}$ & $\mathrm{AO}$ & DE & 00 & (T) & D & A & & 10:40am & & 5 \\
\hline $\mathrm{Pu}$ & F1 & $\mathrm{F} 2$ & 6 & F4 & F5 & F6 & F7 & F8 & PII> & $\mathrm{PII}<$ & $\mathrm{Cr}$ & $\mathrm{AO}$ & DE & 0 & (T) & D & $\mathrm{A}$ & 7 & $10: 41 \mathrm{am}$ & & 6 \\
\hline $\mathrm{Pu}$ & F1 & F2 & 6 & F4 & F5 & F6 & F7 & F8 & PII> & $P I I<$ & $\mathrm{Cr}$ & $\mathrm{AO}$ & DE & (a) & $\mathrm{T}$ & $\mathrm{D}$ & A & 7 & 10:43am & & 7 \\
\hline $\mathrm{Pu}$ & F1 & F2 & F3 & F4 & F5 & F6 & F7 & F8 & $\mid x>$ & $P \|<$ & $\mathrm{Cr}$ & $\mathrm{AO}$ & DE & 00 & (T) & $\mathrm{D}$ & $\mathrm{A}$ & & 10:44am & & 8 \\
\hline $\mathrm{Pu}$ & F1 & F2 & F3 & F4 & F5 & Co & F7 & F8 & $P \|>$ & $P I I<$ & $\mathrm{Cr}$ & $\mathrm{AO}$ & $\mathrm{DE}$ & 00 & (T) & $\mathrm{D}$ & $\mathrm{A}$ & 5* & $10: 48 \mathrm{am}$ & & 9 \\
\hline $\mathrm{Pu}$ & F1 & $\mathrm{F} 2$ & F3 & F4 & F5 & t) & F7 & F8 & $P \|>$ & $P \|<$ & $\mathrm{Cr}$ & $\mathrm{AO}$ & DE & (a) & $\mathrm{T}$ & $\mathrm{D}$ & A & $5^{*}$ & 10:49am & & 10 \\
\hline $\mathrm{Pu}$ & F1 & $\mathrm{F} 2$ & F3 & F4 & F5 & F6 & F7 & F8 & 18 & $\mathrm{PII}<$ & $\mathrm{Cr}$ & $\mathrm{AO}$ & $\mathrm{DE}$ & 0 & (T) & $\mathrm{D}$ & $\mathrm{A}$ & & 10:51am & & 11 \\
\hline$P 6$ & F1 & $\mathrm{F} 2$ & F3 & F4 & F5 & F6 & F7 & F8 & PII> & $P I I<$ & $\mathrm{Cr}$ & $\mathrm{AO}$ & DE & 00 & (T) & $\mathrm{D}$ & A & & & & 12 \\
\hline \multicolumn{14}{|c|}{ Frecuencia \# } & 3 & 8 & & & & & & \\
\hline \multicolumn{22}{|c|}{ Observaciones } \\
\hline \multicolumn{22}{|l|}{1} \\
\hline \multicolumn{22}{|l|}{2} \\
\hline \multicolumn{22}{|l|}{3} \\
\hline 4 & \multicolumn{21}{|c|}{ Cloruro de paladio } \\
\hline \multicolumn{22}{|l|}{5} \\
\hline \multicolumn{22}{|l|}{6} \\
\hline 7 & \multicolumn{21}{|c|}{ Peróxido de hidrógeno } \\
\hline \multicolumn{22}{|l|}{8} \\
\hline \multicolumn{22}{|l|}{9} \\
\hline 10 & \multicolumn{21}{|c|}{ Tecnología de manejo de fluidos, TEM. } \\
\hline \multicolumn{22}{|l|}{11} \\
\hline 12 & & & & & & & & & & & & & & & & & & & & & \\
\hline
\end{tabular}

\section{CONCLUSIONES}

La herramienta de medición y recorrido del trabajo realizado dentro de las bodegas ayuda de visualizar el trabajo desempeñado $y$, por consiguiente, permite encontrar patrones de comportamiento en los desplaza-mientos realizados por los trabajadores. La identificación de malas prácticas y su pronta corrección, representa un insumo importante para la mejora de la productividad; razón por la cual surge el diseño de esta herramienta.

El número de módulos de estantes visitados en el CEMIM, según las mediciones y recorridos analizados, facili-taron la construcción de esta herramienta. Es decir, solo se ocuparon alrededor de 8 a 10 filas del formato presentado con la herra mienta. Así, el campo de observaciones cuenta con el suficiente espacio para especificar el trabajo realizado.

La columna que registra el tiempo, indicado la hora a la que se realizan las actividades, otorgan una visión general de la duración de las actividades. Este ítem no solo indica el tiempo de la duración de la actividad, sino que ubica al analista de la herramienta dentro del tiempo universal coordinado (UTC). Es decir, se puede conocer cuál es la duración del recorrido, así como la hora en la que se realizó esta actividad.

La herramienta también cuenta con la simbología de los diagramas de operaciones: Operación, Transporte, Demoras y Almacenamiento. Estos símbolos 
deben señalarse en la herramienta, de tal forma, que permita calcular la frecuencia de estas actividades durante la visita a las bodegas. Esta frecuencia tiene la ventaja de servir como insumo para la aplicación de técnicas fundamentadas en la filosofía lean manufacturing para la identificación de actividades necesarias y que añaden valor a las operaciones.

Añadir la variable equipo a la herramienta representa una fortaleza en sí misma, debido a que, según el equipo utilizado, el tiempo registrado de las actividades dependerá de la dificultad de maniobrarlas. En este sentido, se puede analizar cuáles equipos afectan los tiempos de duración de las actividades y así, tomar las medidas correspondientes de acuerdo con su utilización.

\section{REFERENCIAS}

[1] R. García, Estudio del trabajo, 2da ed., México: McGraw-Hill, 2005, pp.33-36.

[2] F. Meyers, Estudio de tiempos y movimientos: para la manufactura gil, México: Pearson Education, 2000

[3] C. Dávila, Teorías organizacionales y administración: Enfoque crítico, 2 da ed., Colombia: McGraw-Hill Internamericana S.A., 2001, pp.74-98.

[4] W.J.Hopeand M.L.Spearman, Factory Physics, 3ra ed., Estados Unidos: Waveland Press Inc., 2008, pp. 20-32

[5] F. Morlock, et al., "Teaching Methods-Time Measurement (MTM) for Workplace Design in Learning Factories" in 7th Conference on Learning Factories (CLF 2017), CLF 2017, vol. 9, pp.369-375. DOI: 10.1016/j.promfg.2017.04.033

[6] M. Bejan, "Standarization and some of her artisans", Bulletin Stiintific, pp. 6-14, Junio, 2018.

[7] M. Cardona, "Ingeniería de métodos y Medición del Trabajo: Eficiencia para pequeña industria", Revista M\&M, pp.146-152, Diciembre, 2008.

[8] L. Colligan, et al., "Does the process map influence the outcome of quality improvement work? A comparison of a sequential flow diagram and a hierarchical task analysis diagram", BMC health services research, vol. 10, no. 1, pp.1-
9, Enero, 2010. DOI: 10.1186/1472-6963-10-7.

[9] D. Ivanov, A. Tsipoulanidis, and J. Schonberger, "Layout Planning". In Global Supply Chain and Operations Management, Suiza: Springer, 2016, pp.279-300. DOI: 10.1007/978-3-319-24217-0_10

[10] A. Azadeh, M. Moghaddam, and M. Sheikhalishahi, "Optimization of facility layout design with ambiguity by an efficient fuzzy multivariate approach", The International Journal of Advance Manufacturing Technology, vol. 84, no. 1-4, pp.565-579, Abril, 2016. DOI: 10.1007/s00170-015-7714-x

[11] P. Centobelli, R. Cerchione, T. Murino, and M. Gallo, "Layout and material flow optimization in digital factory", International Journal of Simulation Modelling, vol. 15, no. 2, pp.223-235. DOI: 10.2507/IJSIMM15(2)3.327

[12] C. Huawei, L. Guoping, T. Haining, W. Aimin, and N. Ruxin, "Layout adjustment of cellular production line based on material logistic analysis", The International Journal of Advanced Manufacturing Technology, vol. 87, no. 5-8, pp.1409-1420. DOI 10.1007/s00170-013-4784-5

[13] R. Hernández, C. Fernández, and P. Baptista, Metodología de la Investigación, 6th ed., México D.F.: Mc Graw Hill, 2014, pp. 152-163.

[14] P. Magu, K. Khanna, and P. Seetharaman, "Path process chart - A technique for conducting time and motion study" in 6th International Conference on Applied Human Factors and Ergonomics (AHFE 2015) and the Affiliated Conferences, AHFE 2015, vol. 3, pp.6475-6482. DOI:10.1016/j.promfg.2015.07.929

[15] N. Tarazona, "Estudio de métodos y tiempos en el Centro de Manejo Integral de Muestras (CEMIM) del Instituto Colombiano de Petróleo (ICP) de Ecopetrol", CEMIM, Santander, Tech. Rep., Acuerdo de Cooperación No. 007, 2018.

[16] J.S. Lamus, "Plan de mejoramiento de los pro-cesos de manejo integral de muestras y gestión de residuos peligrosos en las instalaciones del Centro de Manejo Integral de Muestras (CEMIM) del Centro de Innovación y Tecnología ICP", CEMIM, Santander, Tech. Rep., Acuerdo de Cooperación No. 002, 2017. 